\title{
EFFECTIVE LAGRANGIANS FOR QCD: DECONFINEMENT AND CHIRAL SYMMETRY RESTORATION
}

\author{
ÁGNES MÓCSY \\ Institut für Theoretische Physik, J.W. Goethe-Universität, Postfach 111932 \\ 60054 Frankfurt am Main, Germany \\ E-mail: mocsy@th.physik.uni-frankfurt.de \\ FRANCESCO SANNINO* \\ NORDITA 85 The Niels Bohr Institute, Blegdamsvej 172100 \\ Copenhagen O, Denmark \\ * Speaker at the conference. \\ E-mail: francesco.sannino@nbi.dk \\ KIMMO TUOMINEN \\ University of Jyväskylä, Department of Physics, P.O. Box 35, \\ 40014 Jyväskylän Yliopisto, Finland \\ E-mail: kimmo.tuominen@phys.jyu.fi
}

\begin{abstract}
Effective Lagrangians for Quantum Chromodynamics (QCD) especially suited for understanding deconfinement and chiral symmetry restoration at non-zero temperature and matter density are reviewed. These effective theories allow one to study generic properties of phase transitions using non-order parameter fields without loosing the information encoded in the true order parameter. For the pure gauge theory we demonstrate that, near the deconfining phase transition, the center group symmetry is naturally linked to the conformal anomaly. Another relevant outcome is that when the theory contains also quarks we can explain the intertwining of chiral symmetry restoration and deconfinement for QCD with matter fields either in the fundamental or in the adjoint representation of the gauge group. As a test of our general approach we show that our results are applicable also at non-zero baryon chemical potential. We also predict new testable substructures to be present in the phase diagram of quarks in the adjoint representation of the gauge group. Here we provide some new insights on the large $N$ limit of gauge theories by investigating the hadronic world. We propose that the world of infinite $N$ should already be well described when $N=6$ for QCD with two and three light flavors. Finally, we suggest possible future applications of our results for heavy ions collisions.
\end{abstract}




\section{Introduction}

Symmetries, anomalous and exact, are used to constrain effective Lagrangian theories. The latter are applicable to any non perturbative region of the QCD or QCD-like phase diagram, whenever the relevant degrees of freedom and the associated symmetries are known. To decide in which phase a strongly interacting theory can be, one uses experimental inputs, model computations such as Nambu-Jona Lasinio, and/or computer simulations. Exact non perturbative constraints, such as anomaly matching conditions, are another elegant and powerful way to help deciding among the phases that a strongly interacting theory (vector- and chiral-like) can be in. The original idea of t'Hooft has been extended ${ }^{1,2,3}$ to strongly interacting gauge theories at non-zero chemical potential. At non zero temperature anomaly matching conditions are also expected to be applicable. However, here corrections arise to the Ward anomalous identity ${ }^{a}$. 't Hooft anomaly conditions are not sufficient, in general, to select a unique phase. A possible guide at zero chemical potential has been suggested in ${ }^{4}$. Simply, one chooses the phase, respecting anomaly matching conditions, with the lowest number of degrees of freedom counted according to the coefficient of the entropy. This guide works well for known theories, such as QCD with two flavors and three colors, while it allows a number of predictions for strongly interacting gauge theories, such as chiral gauge theories. It would be interesting to investigate a possible generalization to non-zero chemical potential.

Already, a number of novel effective Lagrangians have been written in literature to describe QCD and similar theories at zero temperature (see ${ }^{8}$ for a review). Let us now mention a few before turning to the problem of deconfinement and chiral symmetry breaking.

At zero temperature and quark chemical potential a number of quantitative non-perturbative predictions about the spectrum and the vacuum properties of QCD with one Dirac flavor have been made by constructing

\footnotetext{
${ }^{\text {a The Adler-Bardeen theorem still }}{ }^{5}$ applies but the Sutherland-Veltman one ${ }^{6}$ can be violated when Lorentz symmetry breaks ${ }^{7}$. The modification of the anomalous Ward identity at non-zero temperature is due to a non-local operator induced by temperature corrections computed using the pion Lagrangian ${ }^{7}$. Why this is not a problem at non-zero chemical potential? Take again the pion effective Lagrangian whose coefficients now depend on the baryon chemical potential and image to compute higher order corrections. These corrections are similar to the one in vacuum (actually more suppressed at large chemical potential) no violation of locality is found with no consequence on the Sutherland-Veltamn theorem.
} 
an effective Lagrangian able to interpolate from super Yang-Mills to QCD ${ }^{9}$. These predictions can be tested via standard lattice simulations. Without entering in details we can say that these results are linked to a different type of $1 / N$ expansion around the supersymmetric limit ${ }^{10}$, in which the fermions transform according to the two index antisymmetric representation of the gauge group.

This expansion in the inverse of number of colors may very well be more convergent then the ordinary $1 / N$ expansion. Here the fermions remain in the fundamental representation of the gauge group, while increasing the number of colors. In ${ }^{11}$, for example, the existence of a critical number of colors has been identified. For and above this number of colors the low energy $\pi-\pi$ scattering amplitude, computed from the sum of the current algebra and vector meson terms, is crossing symmetric and unitary at leading order in a $1 / N$ expansion. This critical number of colors turns out to be $N=6$, and is insensitive to the explicit breaking of chiral symmetry. This means that ordinary $1 / N$ corrections for the real world are large. These results are supported by the findings in ${ }^{12}$. As an important outcome, our results are consistent with the expectation that the low lying sigma state, $\sigma(560)$, is not a $q \bar{q}$ object ${ }^{13,14,15,16}$. This has implications on the physics of chiral symmetry restoration, since this state should not be considered as the chiral partner of the pion, as it is at times assumed in literature. While we expect large $1 / N$ corrections for $N=3$, for six or more colors and with two or three flavors the physics should be well described by the large number of colors limit, for which a number of properties can be diagrammatically deduced ${ }^{17}$. $S U(6)$ gauge theories are currently explored by lattice simulations ${ }^{18}$. Encouraged by the findings in the hadronic world $11,12,15$, we predict a transition from the world of infinite $N$ to the world of small $N$ already for $N=6$ with two flavors, and possibly with three flavors as well. Some ideas on $N=$ infinity at non-zero temperature are also available ${ }^{19}$.

Deconfinement and chiral symmetry restoration as function of temperature, quark chemical potential or number of flavors has always attracted much interest ${ }^{20}$. Here we introduce recently developed effective Lagrangians for Quantum Chromodynamics (QCD), relevant for gaining insight on the deconfinement and/or chiral symmetry restoration problem at non-zero temperature and matter density. In literature one can already find a large number of models which try to understand/fit lattice results. The goal we have here is to provide a unifying point of view in which different models can be seen as different description of the same physics. At 
the same time we try to develop new ideas and tools to investigate phase transitions. Clearly, some of the presented tools need to be sharpened in the future.

We first consider the effective theory unifying two apparently very different sectors of a generic Yang-Mills theory at non-zero temperature ${ }^{23}$ : the hadronic sector and the Polyakov loop. This theory is able to communicate the information about the center group symmetry to the hadronic states. It also provides the link between deconfinement and conformal anomaly. The latter has already been used for years in literature for describing the deconfinement phase transition in pure glue.

The basic general idea is to generalize the Landau theory by including non-order parameter fields. Technically, we integrate in a heavy field. We will see that this allows us to study generic properties of phase transitions using the non-order parameters field. Effective Lagrangians for strongly interacting theories with matter fields will also be reviewed here. Via these theories we offer a simple, economical and unifying interpretation of the intertwining between chiral symmetry restoration and deconfinement in QCD with matter fields either in the fundamental, or in the adjoint representation of the gauge group, as function of temperature and/or chemical potential. We also show that the most relevant term is a trilinear interaction between the singlet field and the order parameter. This allowed interaction term differentiates between the different fermion representations, and it has been neglected previously in the literature ${ }^{24,25}$. We finally suggest possible physical applications.

\section{Heavy Fields and Phase Transitions}

The phase transition dynamics and the associated critical behavior are best investigated using order parameters. These are the degrees of freedom whose correlation length diverges when approaching the phase transition. However, the choice of the order parameters is not always obvious. Furthermore, it often turns out that even if the order parameter can be formally constructed, this may be hard, or even impossible to be directly detected experimentally. A time honored example is the Polyakov loop in gauge theories. The characteristic feature of an order parameter is that is zero in the symmetric phase, but attains a finite non-zero value in the symmetry broken phase. In what follows we will term order parameter field a field whose expectation value is a true order parameter, that has the characteristic behavior described above. Any field whose expectation value does 
not have this kind of behavior will be called a non-order parameter field. For simplicity, in the following we will consider non-order parameter fields which are singlets under the symmetry transformations acting on the order parameter.

One can ask some simple questions: Given a system consisting of an order parameter and singlet field(s), what can we learn about the phase transition by monitoring the singlet field? Can we identify the onset of the phase transition without referring to the order parameter? We will show that there is a clear and universal characteristic behavior of the singlet field, induced by the order parameter close to the phase transition. Our considerations are universal, and as such, can be carried over to virtually any phase transition once the symmetries of the order parameter are identified. We will explicitly consider the cases of $Z_{2}$ symmetry, which is of relevance for the pure Yang-Mills gauge theory with two colors, and $S U(4)$ which is of relevance for the effective theories of two color QCD with two quark flavors in either the fundamental or adjoint representation of the gauge group.

Lattice simulations of the pure Yang-Mills gauge theory already confirm our prediction, that the behavior of the order parameter close to the critical temperature is reflected in the behavior of the non-order parameter field.

For QCD with quarks lattice simulations pose the following interesting puzzle: Why, for matter in the fundamental representation deconfinement and chiral symmetry restoration appear to be linked with a single phase transition observed at a given critical temperature, while for matter in the adjoint representation there are two phase transitions, well separated in temperature? We will show how our effective Lagrangian description can offer a simple unifying way of addressing this puzzle.

\section{From the Polyakov Loop to the Glueballs}

\subsection{A Unifying Model: Center Group Symmetry and Conformal Anomaly}

Consider the pure Yang-Mills theory with $S U(N)$ gauge symmetry. At non-zero temperature the Yang-Mills theory possesses a global $Z_{N}$ symmetry. This symmetry is intact at low temperatures and is broken at high temperatures. The associated order parameter is the Polyakov loop, which is the trace of the thermal Wilson line. Under the action of $Z_{N}$ the Polyakov loop transforms as $\ell \rightarrow z \ell$ with $z \in Z_{N}$.

The Polyakov loop condensation is associated to deconfinement. The reason being that the potential $V(\vec{x}, T)$ at a given temperature $T$ between 
static fundamental charges is related to the Polyakov loop:

$$
\exp (-V(\vec{x}, T) / T) \equiv\left\langle\ell(\overrightarrow{0}) \ell^{\dagger}(\vec{x})\right\rangle \underset{|\vec{x}| \rightarrow \infty}{\longrightarrow}|\ell|^{2} \quad,
$$

For $T<T_{c}$ the static quarks experience a linearly rising potential

$$
V(\vec{x}, T)=\sigma(T)|x|,
$$

where $\sigma(T)$ is the string tension. For $T>T_{c}$ the potential is no longer a confining one:

$$
V(\vec{x}, T) \propto k(T),
$$

and the potential does not depend on the distance between the static sources, here chosen to be in the fundamental representation of the gauge group.

Close to $T_{c}$, using age-old arguments by Landau, one can write a mean field effective potential of the form

$$
V(\ell)=T^{4} \mathcal{F}(\ell),
$$

where $\mathcal{F}(\ell)$ is a $Z_{N}$-invariant polynomial in $\ell$. It was Svetitsky and Yaffe who provided the first reliable non-perturbative study of the critical behavior of Yang-Mills theories as well as the form of the effective potential displayed above ${ }^{26,27,28}$. The idea of describing the Yang-Mills pressure using directly a mean field theory of Polyakov loops has been recently advocated by Pisarski ${ }^{29}$. This model has since been used in several phenomenological studies ${ }^{24,30}$, which miss to include of a relevant term ${ }^{31,32,33}$. The scale dimension is set by the temperature. The physical states (i.e. the hadrons), however, do not carry any charge under $Z_{N}$. So in order for a hadronic state to know about the center group symmetry this will have to communicate with the Polyakov loop. Besides, approaching the critical temperature from the confining side, the relevant degrees of freedom are the hadronic states which for the pure Yang-Mills theory are the glueballs.

In the following, we consider as important representative of the hadronic spectrum the lightest scalar glueball. At zero temperature the well known effective theory constrained by the trace anomaly has been constructed in ${ }^{34}$, using the potential of the form

$$
H \ln \frac{H}{\Lambda^{4}} .
$$

The glueball field $H$ is related to $G_{\mu \nu}^{a} G^{\mu \nu, a}$, where $G^{\mu \nu, a}$ is the gluon field strength and $a=1, \ldots, N^{2}-1$ the gauge indices. It has been shown that this potential encodes the basic properties of the Yang-Mills vacuum at 
$T=0^{35}$, and it has also been used at non-zero density ${ }^{36}$ and temperature 37,38. As we increase the temperature the Polyakov loop becomes a well defined object. We stress that sofar in the literature the Yang-Mills pressure has been described by either glueball theories ${ }^{37}$ and their generalizations ${ }^{39}$, or directly with the Polyakov loop model ${ }^{24}$. In effective glueball theories a possible drop at $T_{c}$ of the non-perturbative contribution to the gluon condensate has been often considered as an indication of deconfinement ${ }^{37,36}$. To be more precise, since temperature breaks Lorentz invariance, one should consider independently the non-perturbative contribution to the trace anomaly of $G^{0 i} G_{0 i}$ and $G^{i j} G_{i j}$. In the vacuum we know that the expectation value of the second component dominates since confinement is associated to condensation of magnetic type charges. It is this component which should decrease at the phase transition. However, since the gluon condensate is not an order parameter for the center group symmetry there is, a priori, no guarantee that such a drop, even if observed, should appear at the same critical temperature. Another important point is that the glueball theory by construction is blind to the number of colors. This explains why it always predicts a first order phase transition independent of the number of colors of the underlying Yang-Mills theory one wants to describe ${ }^{30}$.

The Polyakov model, on the other hand, has automatically built in the knowledge about the number of colors, but looses contact with a simple physical picture in terms of hadronic states.

We will now show, by marrying these two theories, that: i) The drop of the non-perturbative part of the gluon condensate must happen exactly at the deconfining phase transition ${ }^{23}$, partially justifying the glueball models; ii) The information about the order of the transition is now encoded in a nontrivial way in the profile of the condensate drop.

We start with the following general potential constructed using all of the relevant symmetries, i.e. trace anomaly, $Z_{N}$ invariance and analyticity of the interaction term between the glueball field and the Polyakov loop ${ }^{23}$

$$
V(\ell, H)=H \ln \frac{H}{\Lambda^{4}}+V_{T}(H)+H \mathcal{P}(\ell)+T^{4} \mathcal{F}(\ell) .
$$

Here $V_{T}(H)$ is the intrinsic temperature dependence of the glueball gas, which we can neglect in what follows, since the glueball is heavy near the phase transition. The most general interaction term compatible with the saturation of the trace anomaly is $H \mathcal{P}(\ell)$, where $\mathcal{P}(\ell)$ is a $Z_{N}$ symmetric polynomial in $\ell$. In the static limit we can use the equation of motion for the $H$ field to integrate this out in terms of $\ell$ field, yielding the functional relation $H \equiv H(\ell)$. This shows that the center group symmetry is 
transferred to the singlet sector of the theory. However, we find it more illuminating to keep both fields. For two colors we take

$$
\begin{aligned}
& \mathcal{F}(\ell)=a_{1} \ell^{2}+a_{2} \ell^{4}+\mathcal{O}\left(\ell^{6}\right), \\
& \mathcal{P}(\ell)=b_{1} \ell^{2}+\mathcal{O}\left(\ell^{4}\right)
\end{aligned}
$$

and the coefficients $a_{2}$ and $b_{1}$ are assumed to be positive and independent of the temperature, while the coefficient $a_{1}$ is taken to be $a_{1}(T)=\alpha\left(T-T_{*}\right)$ with $\alpha>0$. Using these expressions in the potential and solving for the minimum, one finds that below $T_{c}$ the two fields are completely decoupled and

$$
\langle\ell\rangle=0, \quad\langle H\rangle=\Lambda^{4} / e .
$$

As the critical temperature $T_{c}=T_{*}+\frac{b_{1}}{e \alpha} \frac{\Lambda^{4}}{T_{c}^{3}}$ is reached, the symmetry is spontaneously broken and the Polyakov loop obtains a non-zero expectation value, which affects also the one for $H$ :

$$
\langle\ell\rangle \propto \frac{\Delta T}{T_{c}}, \quad\langle H\rangle=\frac{\Lambda^{4}}{e} \exp \left[-2 b_{1}\langle\ell\rangle^{2}\right] .
$$

For three colors the essential modification is the inclusion of the $\ell^{3}$ and $\ell^{* 3}$ terms into the Polyakov loop potential $\mathcal{F}$, while the interaction potential becomes $\mathcal{P} \propto|\ell|^{2}$. The cubic terms render the transition first order, and thus the change in both the Polyakov loop and the glueball becomes discontinuous. Once more we deduce that the change in the order parameter $\ell$ induces a change in the expectation value of the non-order parameter field. The behavior in these two cases is shown in figure 1 . We then conclude
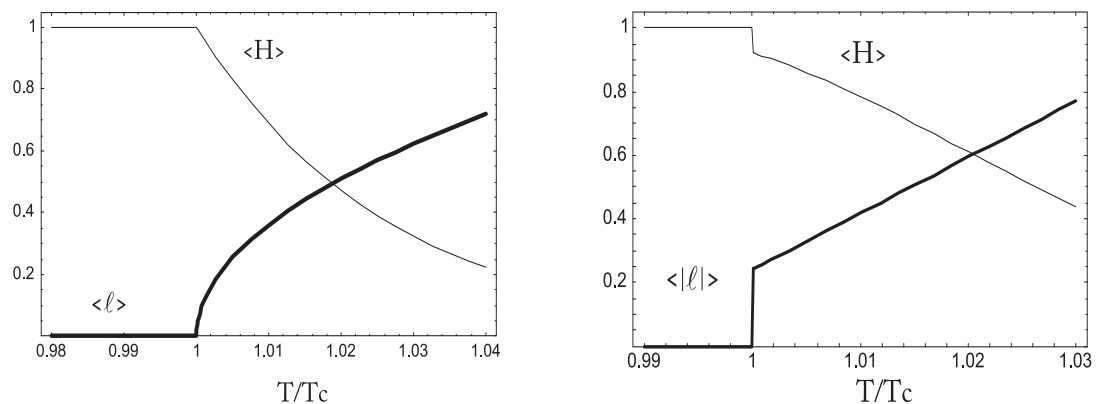

Figure 1. The behavior of the expectation values of the fields as a function of temperature. The left panel shows the two color case with second order transition. The right panel shows the three color case with the first order transition, characterized by a finite discontinuity in the expectation values. 
that, not only the drop of the non-perturbative part of the gluon condensate knows about the Yang-Mills phase transition, but also that the drop occurs at $T_{c}$. Furthermore, the profile of the gluon condensate as function of temperature encodes the information on the order of the phase transition.

This theory unifies two apparently very different pictures of the deconfining phase transition.

\subsection{Beyond the Static Limit: A Renormalizable Approach}

Now we will improve on some of the approximations made in the previous section. We consider fluctuating fields and hence we include the kinetic terms. Furthermore, in order to keep the discussion as general as possible we will not confine ourselves to the Yang-Mills theory. We start with a renormalizable Lagrangian for two real scalar fields constrained only by the symmetries and renormalizability. We consider the case of an exact $Z_{2}$ symmetry. The generalization to a $Z_{N}$ or an $O(N)$ symmetry is straightforward. The order parameter field is $\chi$ which transforms according to $\chi \rightarrow z \chi$ with $z \in Z_{2}$. We will consider two cases: A time independent, $\chi=\chi(x)$, and a time dependent one, $\chi=\chi(t, x)$. The former being relevant for deconfinement in QCD when identified with $\ell$, while the latter can be of use if identified with the electroweak Higgs field for example. The most general potential, in both cases restricted by symmetry and renormalizability, is ${ }^{\mathrm{b}}$ :

$$
V(h, \chi)=\frac{m_{h}^{2}}{2} h^{2}+\frac{m_{\chi}^{2}}{2} \chi^{2}+\frac{\lambda}{4 !} \chi^{4}+\frac{g_{1}}{2} h \chi^{2}+\frac{g_{2}}{4} h^{2} \chi^{2}+\frac{g_{3}}{3 !} h^{3}+\frac{g_{4}}{4 !} h^{4}
$$

This potential can be considered as a truncated version of the full glueball theory of the previous section, after having defined $H=\langle H\rangle(1+$ $h /(\sqrt{c}\langle H\rangle))$ and $\chi=\sqrt{\kappa} \ell$. Consider first the case of time-independent order parameter, $\chi=\chi(x)$. In this case only the zero mode of $h$ is relevant and the Lagrangian for the three dimensional theory is

$$
\begin{aligned}
-\mathcal{L}_{3 D} & =\frac{1}{2} \nabla h \nabla h+\frac{1}{2} \nabla \chi \nabla \chi+\frac{1}{2} m_{h}^{2} h^{2}+\frac{1}{2} m_{\chi}^{2} \chi^{2} \\
& +T \frac{\lambda}{4 !}\left(\chi^{2}\right)^{2}+\sqrt{T} \frac{g_{1}}{2} h \chi^{2}+T \frac{g_{2}}{4} h^{2} \chi^{2}+\sqrt{T} \frac{g_{3}}{3 !} h^{3}+T \frac{g_{4}}{4 !} h^{4} .
\end{aligned}
$$

We are interested in evaluating the corrections to the mass of the singlet field $h$ induced by the fluctuations of the order parameter. These are dom-

${ }^{\mathrm{b}}$ There is also a linear term in $h$ whose effects up to one loop have been studied in detail in ${ }^{32}$ and are shown not to affect our results. 
inated at one loop by the diagram

$$
\bigcirc-T\left(\frac{g_{1}}{2}\right)^{2} \int \frac{d^{3} k}{(2 \pi)^{3}} \frac{1}{\left(k^{2}+m_{\chi}^{2}\right)^{2}}=T \frac{g_{1}^{2}}{32 \pi m_{\chi}} \text {. }
$$

Due to the infrared singularity this one-loop result breaks down at $T_{c}$. On general grounds though, discussed in detail in ${ }^{32}$, we expect the mass of $h$ to stay finite. Therefore, further improvements are required. In order to determine the fixed points and the associated critical exponents we can use the $4-\epsilon$ analysis. This is currently under investigation ${ }^{40}$. Here, however, we shall present a simple resummation scheme which is exact in the case of the $O(N)$ symmetric theory in the large $N$ limit. Nota bene, that although we expect this simple model to be at best only qualitatively correct, as we shall see, it reproduces the general features of rather "old" data ${ }^{41}$ surprisingly well.

The finite result is given by the sum of simple bubble diagrams, yielding for the mass for the singlet field at $T_{c}$

$$
m_{h}^{2}(T)=m_{h}^{2}-T \frac{g_{1}^{2}}{16 \pi m_{\chi}+\lambda T} \stackrel{T \rightarrow T_{c}}{\longrightarrow} m_{h}^{2}-\frac{g_{1}^{2}}{\lambda} .
$$

A similar bubble sum can be performed in the broken phase with the replacement $m_{\chi}^{2} \rightarrow 2\left|m_{\chi}\right|^{2}$, leading to the same value for the mass of the singlet field at $T_{c}$. The critical behavior can be traced via the slope of the screening mass, which is simply

$$
\mathcal{D}^{ \pm} \equiv \lim _{T \rightarrow T_{c}^{ \pm}} \frac{1}{\Delta m_{h}^{2}\left(T_{c}\right)} \frac{d \Delta m_{h}^{2}(T)}{d T} \sim t^{\nu / 2-1},
$$

where $\Delta m_{h}^{2} \equiv m_{h}^{2}(T)-m_{h}^{2}$ and the result is exact only in the limit of large $N$. It is interesting to note that there exists other classes of diagrams which can be exactly resummed, and allow us to depart from the large $N$ limit. In the broken phase such class is given by the diagrams

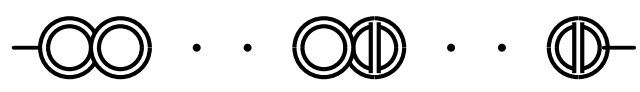

These can be resummed exactly ${ }^{32}$ yielding

$$
m_{h}^{2}(T)=m_{h}^{2}-\frac{g_{1}^{2} \mathcal{I}}{2} \frac{1+\frac{\lambda}{3} \mathcal{I}}{1+\frac{\lambda}{2} \mathcal{I}+\frac{\lambda^{2}}{6} \mathcal{I}^{2}},
$$

where

$$
\mathcal{I}=\frac{T}{8 \pi \sqrt{2}\left|m_{\chi}\right|}
$$


At the critical temperature, once again we find $m_{h}^{2}\left(T_{c}\right)=m_{h}^{2}-\frac{g_{1}^{2}}{\lambda}$. The qualitative behavior of the screening mass is shown in figure 2 . On the
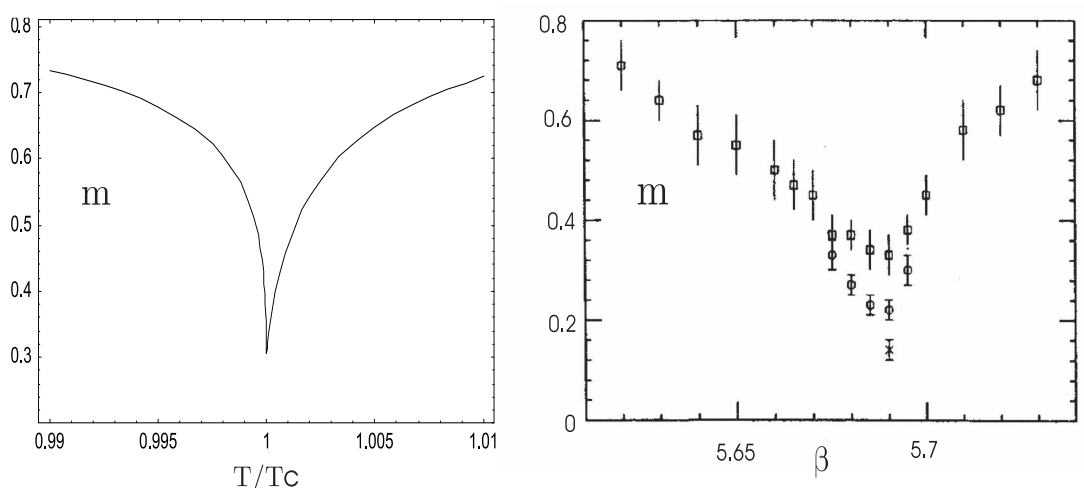

Figure 2. Left panel: mass of the singlet field near $T_{c}$ evaluated using (10) and (12). For this plot we chose $g_{1}^{2} /\left(m^{2} \lambda\right) \sim 0.9$ and $m_{\chi}^{2} /\left[\left(T_{c}-T\right) T\right] \sim 0.004$. Right panel: Lattice data from ${ }^{41}$

left panel is our schematic plot. On the right panel is lattice data from ${ }^{41}$ showing the behavior of the scalar glueball screening mass in pure $\mathrm{SU}(3)$ gauge theory. Such drop in the glueball screening mass has been further identified in ${ }^{42}$ for both $\mathrm{SU}(2)$ and $\mathrm{SU}(3)$.

In the case of the time-dependent order parameter field, $\chi=\chi(t, x)$, all the modes contribute to the analysis at finite temperature. We have shown ${ }^{32}$ that the pole mass of the singlet field is not affected by the phase transition, while the screening mass is still IR sensitive. Furthermore, the screening mass of the singlet field shows the same behavior as described above.

We stress that the presence of the trilinear term $h \chi^{2}$ plays a vital role and should not be neglected.

\section{Chiral Symmetry versus Confinement}

Consider now adding quarks into the Yang-Mills theory. Lattice results for quarks in the fundamental representation of the gauge group show a clear indication that as chiral symmetry is restored, the expectation value of the Polyakov loop rises, signalling deconfinement. Figure 3 from ${ }^{43}$ illustrates 

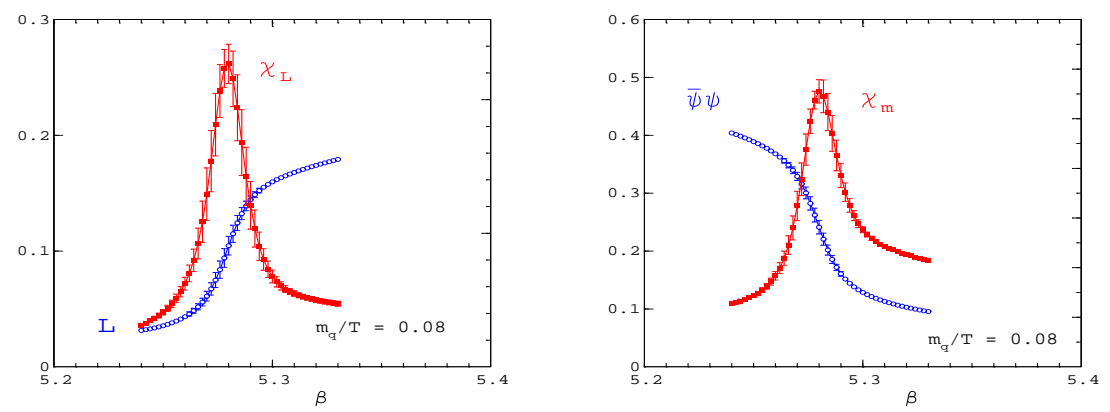

Figure 3. Behavior of the Polyakov loop (left panel) and of the chiral condensate (right panel) and the corresponding susceptibilities, as determined on the lattice with quarks in the fundamental representation. From ${ }^{43}$.

that the critical temperatures ${ }^{\mathrm{c}}$ of the deconfinement and of the chiral symmetry restoration coincide, $T_{\text {chiral }}=T_{\text {deconf }}$. We also learn from lattice results that for quarks in the adjoint representation this is not the case, as clearly indicated in Fig. 4 taken from ${ }^{44}$. Here the critical temperature for
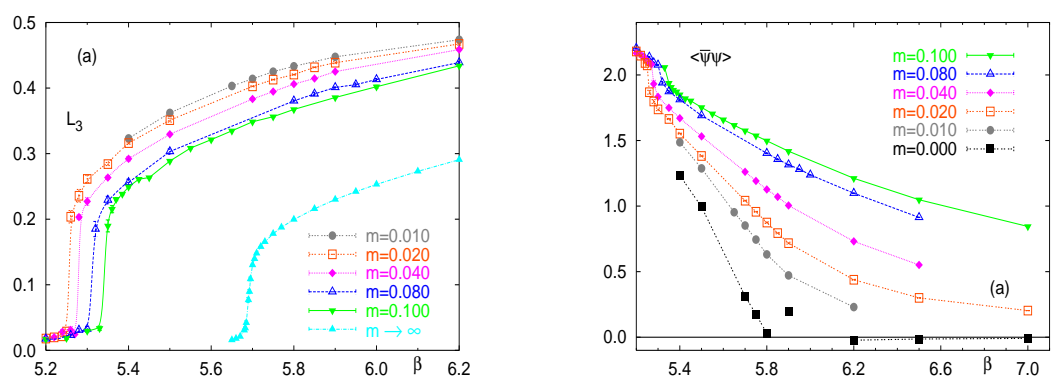

Figure 4. Behavior of the Polyakov loop (left panel) and of the chiral condensate (right panel) as determined on the lattice with quarks in the adjoint representation. From ${ }^{44}$.

chiral symmetry restoration is $T_{\text {chiral }} \simeq 8 T_{\text {deconf }}$. Note also, that even if the two transitions happen separately the chiral condensate knows about deconfinement, as the jump in its behavior at $T_{\text {deconf }}$ seems to indicate.

Lattice simulations are already available for two color QCD at non-zero baryon chemical potential, and observe deconfinement for 2 color QCD

${ }^{\mathrm{c}}$ The (pseudo) critical temperatures are identified from the peak position of the corresponding susceptibilities, also shown in figure 3. 
and 8 continuum flavors at $\mu \neq 0$ One of the relevant points is that the

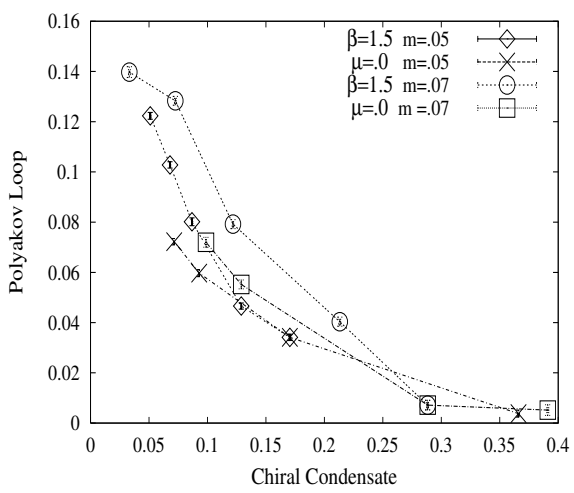

Figure 5. Polyakov loop versus chiral condensate for two color QCD and 8 continuum flavors at non-zero baryon chemical potential as determined on the lattice. From ${ }^{45}$.

Polyakov loop rises when the chiral condensate vanishes, and the two phase transitions happen at the same value of the chemical potential i.e. $\mu_{\text {chiral }}=$ $\mu_{d}{ }^{45}$

Our goal is to provide a simple, economical and unified way to describe all of these features. This is possible thanks to a crucial interaction term which has been neglected in phenomenological investigations ${ }^{24}$, and also in explanations of the coincidence of chiral and deconfining phase transition 25 .

\subsection{General Observations}

The quark representation with respect to the gauge group plays an important role ${ }^{46}$. In fact, when quarks are in the fundamental representation of the gauge group the $Z_{N}$ symmetry is no longer exact for any finite value of the quark mass. When, on the other hand, quarks are in the adjoint representation of the gauge group the center group symmetry is intact.

Chiral symmetry is explicitly broken by a mass term for quarks. In the non-perturbative regime we do not know d the amount of $Z_{N}$ breaking, which is due to introduction of quarks in the fundamental representation.

$\overline{\mathrm{d} \text { Unless a fit to lattice data }{ }^{47} \text { is performed }}{ }^{48}$. 
Therefore we cannot establish which symmetry is more broken for a given quark mass.

Fortunately, at least theoretically, we can take limits in the QCD parameter space, allowing us to make exact statements independently of the lattice results. The first limit we consider is the one in which chiral symmetry is exact, i.e. quark masses are zero. For any number of flavors and colors, $Z_{N}$ is not a symmetry and the only order parameter is the chiral condensate $(\bar{q} q \sim \sigma)$. We will demonstrate in the following sections, that due to the presence of a relevant $Z_{N}$ symmetry breaking term the Polyakov loop ${ }^{\mathrm{e}}$ has an induced critical behavior driven by the chiral phase transition. This term is the most relevant term differentiating among fermion representations. Unfortunately, previously it has been omitted in the literature 24,30 . We expect that its inclusion drastically affects phenomenological predictions and their implications for heavy ion collision experiments.

If the $Z_{N}$ symmetry is only softly broken one might expect another almost phase transition for deconfinement $49,50,51$. The main motivation for having advocated, in the past, two independent phase transitions is related to the possibility of two independent scales in QCD: One associated to chiral symmetry breaking and the other associated to deconfinement. The underlying origin of two different scales is however unclear. Besides, as explained above, lattice results now dismiss this possibility for QCD. A two phase transitions situation is still possible in QCD with zero quark masses in the limit of large number of colors for fixed number of flavors, i.e. $N_{f} / N \ll 1$, but it is unnatural in the case $N_{f} \sim N$.

When turning on the quark masses the situation is less transparent. If the quark masses are smaller than the confining scale of the theory and barring accidental dynamical suppression we expect that by comparing the following ratios

$$
m_{\text {quark }} / \Lambda \text { chiral breaking and } N_{f} / N \quad \mathrm{Z}_{\mathrm{N}} \text { breaking, }
$$

we can have a rough estimate of which symmetry is mostly restored. It might be more accurate to use as ratio for chiral symmetry $m_{\pi} / 4 \pi F_{\pi}$. Note that we expect the $Z_{N}$ breaking terms to be a more complicated function of number of flavors, colors and quark masses $f\left(N_{f}, N_{c}, m_{\text {quark }} / \Lambda\right)$. Near the chiral limit we can clearly drop the quark mass dependence.

\footnotetext{
${ }^{\mathrm{e}}$ Nota bene, that we named the canonically normalized field associated with the Polyakov loop $\chi$. Any quantity having a subscript $\chi$ is associated to the Polyakov loop and not to chiral symmetry. Chiral symmetry related quantities have the subscript $\sigma$.
} 
The situation for the fermions in the adjoint representation of the gauge group will be studied below in some detail along with the study of quarks in the fundamental representation and in the limit of exact chiral symmetry.

We have chosen, in order to illustrate our results, to study the two color theory with $N_{f}$ flavors. The rational behind this choice is that at the same time and with a minor modification of the effective Lagrangian we can discuss the theory with quarks in the fundamental and adjoint representation at non-zero temperature or quark chemical potential. The generalization to three colors is straightforward at non-zero temperature while a bit more involved for the quark chemical potential.

\subsection{Fundamental Representation}

The global quantum symmetry group for two flavors and two colors is $S U\left(2 N_{f}\right)$. After chiral symmetry breaking has occurred, $S U\left(2 N_{f}\right) \rightarrow$ $S p\left(2 N_{f}\right)$, the degrees of freedom in the chiral sector of the effective theory are $2 N_{f}^{2}-N_{f}-1$ Goldstone fields $\pi^{a}$, and a scalar field $\sigma$. For $N_{f}=2$ the potential is 52,53 :

$$
V_{\mathrm{ch}}\left[\sigma, \pi^{a}\right]=\frac{m^{2}}{2} \operatorname{Tr}\left[M^{\dagger} M\right]+\lambda_{1} \operatorname{Tr}\left[M^{\dagger} M\right]^{2}+\frac{\lambda_{2}}{4} \operatorname{Tr}\left[M^{\dagger} M M^{\dagger} M\right]
$$

with $2 M=\sigma+i 2 \sqrt{2} \pi^{a} X^{a}, a=1, \ldots, 5$ and $X^{a} \in \mathcal{A}(S U(4))-\mathcal{A}(S p(4))$. The generators $X^{a}$ are provided explicitly in equation (A.5) and (A.6) of ${ }^{52}$. The Polyakov loop is treated as a heavy field singlet under the chiral symmetry. Its contribution to the potential in the absence of the $Z_{2}$ symmetry is

$$
V_{\chi}[\chi]=g_{0} \chi+\frac{m_{\chi}^{2}}{2} \chi^{2}+\frac{g_{3}}{3} \chi^{3}+\frac{g_{4}}{4} \chi^{4} .
$$

The field $\chi$ represents the Polyakov loop itself. To complete the effective theory we introduce interaction terms allowed by the chiral symmetry

$$
V_{\mathrm{int}}\left[\chi, \sigma, \pi^{a}\right]=\left(g_{1} \chi+g_{2} \chi^{2}\right) \operatorname{Tr}\left[M^{\dagger} M\right]=\left(g_{1} \chi+g_{2} \chi^{2}\right)\left(\sigma^{2}+\pi^{a} \pi^{a}\right) .
$$

The $g_{1}$ term plays a fundamental role and it is exactly the one we already mentioned that have been dropped in previous investigations ${ }^{24,30}$. In the phase with $T<T_{c \sigma}\left(T_{c \sigma} \equiv T_{\text {chiral }}\right)$, where chiral symmetry is spontaneously broken, $\sigma$ acquires a non-zero expectation value, which in turn induces a modification also for $\langle\chi\rangle$. The usual choice for alignement is in the $\sigma$ 
direction, i.e. $\langle\pi\rangle=0$. The extremum of the linearized potential is at

$$
\begin{array}{ll}
\langle\sigma\rangle^{2} \simeq-\frac{m_{\sigma}^{2}}{\lambda}, & m_{\sigma}^{2} \simeq m^{2}+2 g_{1}\langle\chi\rangle, \\
\langle\chi\rangle \simeq \chi_{0}-\frac{g_{1}}{m_{\chi}^{2}}\langle\sigma\rangle^{2}, & \chi_{0} \simeq-\frac{g_{0}}{m_{\chi}^{2}},
\end{array}
$$

where $\lambda=\lambda_{1}+\lambda_{2}$. The previous formulae hold near the phase transition where $\sigma$ is small. Here $m_{\sigma}^{2}$ is the full coefficient of the $\sigma^{2}$ term in the treelevel Lagrangian which, due to the coupling between $\chi$ and $\sigma$, also depends on $\langle\chi\rangle$. Spontaneous chiral symmetry breaking appears for $m_{\sigma}^{2}<0$. In this regime the positive mass squared of the $\sigma$ is $M_{\sigma}^{2}=2 \lambda\left\langle\sigma^{2}\right\rangle$. Near the critical temperature the mass of the order parameter field is assumed to posses the generic behavior $m_{\sigma}^{2} \sim\left(T-T_{\mathrm{c}}\right)^{\nu}$. Equation (19) shows that for $g_{1}>0$ and $g_{0}<0$ the expectation value of $\chi$ behaves oppositely to that of $\sigma:$ As the chiral condensate starts to decrease towards chiral symmetry restoration, the expectation value of the Polyakov loop starts to increase, signaling the onset of deconfinement. This is illustrated in the left panel of figure 6. Positivity of the expectation values implies $2 g_{1}^{2}-\lambda m_{\chi}^{2}<0$, which also makes the extremum a minimum. At the one-loop level one can show ${ }^{32}$ that also $\chi_{0}$ acquires a temperature dependence. When applying the analysis presented in ${ }^{31,32}$, the general behavior of the spatial two-point correlator of the Polyakov loop can be determined. Near the transition point, in the broken phase, the $\chi$ two-point function is dominated by the infrared divergent $\sigma$-loop. This is so, because the $\pi^{a}$ Goldstone fields couple only derivatively to $\chi$, and thus decouple. We find a drop in the screening mass of the Polyakov loop at the phase transition. When approaching the transition from the unbroken phase the Goldstone fields do not decouple, but follow the $\sigma$, resulting again in the drop of the screening mass of the Polyakov loop (this is actually the string tension) close to the phase transition. Consider the variation of the $\chi$ mass near the phase transition with respect to the tree level mass, $m_{\chi}$, defined above the chiral phase transition, $\Delta m_{\chi}^{2}(T)=$ $m_{\chi}^{2}(T)-m_{\chi}^{2}$. Using a large $N$ framework motivated resummation ${ }^{32}$ we deduce:

$$
\begin{array}{ll}
\Delta m_{\chi}^{2}(T)=-\frac{2 g_{1}^{2}\left(1+N_{\pi}\right)}{8 \pi m_{\sigma}+\left(1+N_{\pi}\right) 3 \lambda}, & T>T_{\mathrm{c} \sigma} \\
\Delta m_{\chi}^{2}(T)=-\frac{2 g_{1}^{2}}{8 \pi M_{\sigma}+3 \lambda}, & T<T_{\mathrm{c} \sigma} .
\end{array}
$$

From the above equations one finds that the screening mass of the Polyakov loop is continuous and finite at $T_{\mathrm{c} \sigma}$, and $\Delta m_{\chi}^{2}\left(T_{\mathrm{c} \sigma}\right)=-2 g_{1}^{2} /(3 \lambda)$, indepen- 
dent of $N_{\pi}$, the number of pions. This analysis is not restricted to the chiral/deconfining phase transition. The entanglement between the order parameter (the chiral condensate) and the non-order parameter field (the Polyakov loop) is universal.

\subsection{Adjoint Representation}

As a second application, consider two color QCD with two massless Dirac quark flavors in the adjoint representation. Here the global symmetry is $S U\left(2 N_{f}\right)$ which breaks via a bilinear quark condensate to $O\left(2 N_{f}\right)$. The number of Goldstone bosons is $2 N_{f}^{2}+N_{f}-1$. We take $N_{f}=2$. There are two exact order parameter fields: the chiral $\sigma$ field and the Polyakov loop $\chi$. Since the relevant interaction term $g_{1} \chi \sigma^{2}$ is now forbidden, one might expect no efficient information transfer between the fields. While respecting general expectations the following analysis suggests the presence of a new and more elaborated structure which lattice data can clarify in the near future. The chiral part of the potential is given by (15) with $2 M=\sigma+i 2 \sqrt{2} \pi^{a} X^{a}, a=1, \ldots, 9$ and $X^{a} \in \mathcal{A}(S U(4))-\mathcal{A}(O(4)) . X^{a}$ are the generators provided explicitly in equation (A.3) and (A.5) of 52 . The now $Z_{2}$ symmetric potential for the Polyakov loop is

$$
V_{\chi}[\chi]=\frac{m_{0 \chi}^{2}}{2} \chi^{2}+\frac{g_{4}}{4} \chi^{4}
$$

and the only interaction term allowed by symmetries is

$$
V_{\text {int }}[\chi, \sigma, \pi]=g_{2} \chi^{2} \operatorname{Tr}\left[M^{\dagger} M\right]=g_{2} \chi^{2}\left(\sigma^{2}+\pi^{a} \pi^{a}\right) .
$$

The effective Lagrangian does not know which transition happens first but this is irrelevant for the validity of our general results. Consider the physical case in which the deconfinement phase transition happens first. Denoting the deconfining critical temperature with $T_{\mathrm{c} \chi}$ and the chiral critical temperature with $T_{\mathrm{c} \sigma}$, we put ourselves in the limit $T_{\mathrm{c} \chi} \ll T_{\mathrm{c} \sigma}$. For $T_{\mathrm{c} \chi}<T<T_{\mathrm{c} \sigma}$ both symmetries are broken, and the expectation values of the two order parameter fields are linked to each other:

$$
\begin{aligned}
& \langle\sigma\rangle^{2}=-\frac{1}{\lambda}\left(m^{2}+2 g_{2}\langle\chi\rangle^{2}\right) \equiv-\frac{m_{\sigma}^{2}}{\lambda}, \\
& \langle\chi\rangle^{2}=-\frac{1}{g_{4}}\left(m_{0 \chi}^{2}+2 g_{2}\langle\sigma\rangle^{2}\right) \equiv-\frac{m_{\chi}^{2}}{g_{4}} .
\end{aligned}
$$

The coupling $g_{2}$ is taken to be positive. The expected behavior of $m_{\chi}^{2} \sim$ $\left(T-T_{\mathrm{c} \chi}\right)^{\nu_{\chi}}$ and $m_{\sigma}^{2} \sim\left(T-T_{\mathrm{c} \sigma}\right)^{\nu_{\sigma}}$ near $T_{\mathrm{c} \chi}$ and $T_{\mathrm{c} \sigma}$, respectively, combined with the result of eq. (24), yields in the neighborhood of these two 

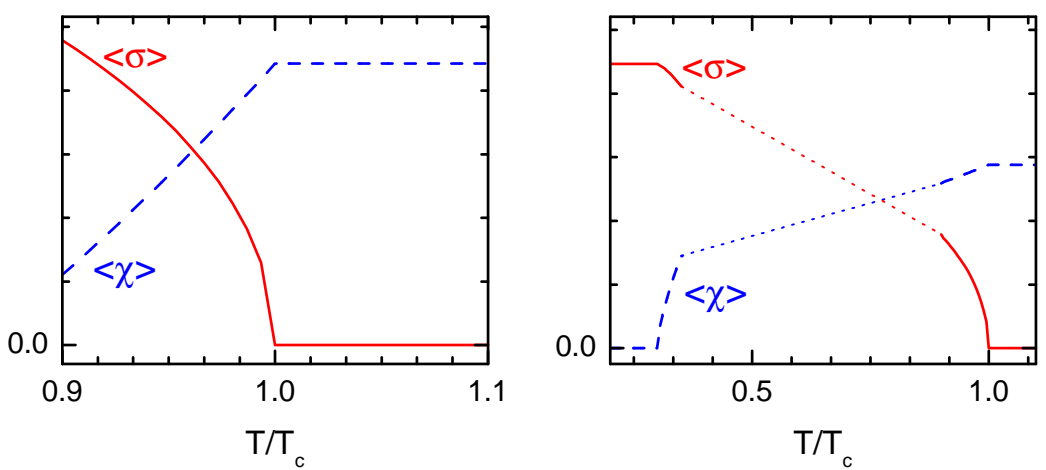

Figure 6. Left panel: Behavior of the expectation values of the Polyakov loop and chiral condensate close to the chiral phase transition as a function of temperature, with massless quarks in the fundamental representation. Right panel: Same as in left panel, for massless quarks in the adjoint representation and $T_{\mathrm{c} \chi} \ll T_{\mathrm{c} \sigma}$ (see discussion in the text).

transitions the qualitative situation illustrated in the right panel of figure 6. On both sides of $T_{\mathrm{c} \chi}$ the relevant interaction term $g_{2}\langle\sigma\rangle \sigma \chi^{2}$ emerges, leading to a one-loop contribution to the static two-point function of the $\sigma$ field $\propto\langle\sigma\rangle^{2} / m_{\chi}$. Near the deconfinement transition $m_{\chi} \rightarrow 0$ yielding an infrared sensitive screening mass for $\sigma$. Similarly, on both sides of $T_{\mathrm{c} \sigma}$ the interaction term $\langle\chi\rangle \chi \sigma^{2}$ is generated, leading to the infrared sensitive contribution $\propto\langle\chi\rangle^{2} / m_{\sigma}$ to the $\chi$ two-point function. We conclude, that when $T_{\mathrm{c} \chi} \ll T_{\mathrm{c} \sigma}$, the two order parameter fields, a priori unrelated, do feel each other near the respective phase transitions. It is important to emphasize that the effective theory works only in the vicinity of the two phase transitions. Interpolation through the intermediate temperature range is shown by dotted lines in the right panel of figure 6 . Possible structures here must be determined via first principle lattice calculations. The infrared sensitivity leads to a drop in the screening masses of each field in the neighborhood of the transition of the other, which becomes critical, namely of the $\sigma$ field close to $T_{\mathrm{c} \chi}$, and of the $\chi$ field close to $T_{\mathrm{c} \sigma}$. The resummation procedure outlined in the previous section predicts again a finite drop for the masses:

$$
\Delta m_{\chi}^{2}\left(T_{\mathrm{c} \sigma}\right)=-\frac{8 g_{2}^{2}\langle\chi\rangle^{2}}{3 \lambda}, \quad \Delta m_{\sigma}^{2}\left(T_{\mathrm{c} \chi}\right)=-\frac{8 g_{2}^{2}\langle\sigma\rangle^{2}}{3 g_{4}} .
$$

We thus predict the existence of substructures near these transitions, when considering fermions in the adjoint representation. Searching for such hidden behaviors in lattice simulations would help to further understand the 
nature of phase transitions in QCD.

\subsection{Quark Chemical Potential}

The analysis can be extended for phase transitions driven by a chemical potential. In fact, for two color QCD this is straightforward to show. When considering fermions in the pseudoreal representation there is a phase transition from a quark-antiquark condensate to a diquark condensate ${ }^{54}$. We hence predict, in two color QCD, that when diquarks form for $\mu=m_{\pi}$, the Polyakov loop also feels the presence of the phase transition exactly in the same manner as it feels when considering the temperature driven phase transition. Such a situation is supported by recent lattice simulations ${ }^{45}$.

\section{Conclusions and Outlook}

We have shown how deconfinement (i.e. a rise in the Polyakov loop) is a consequence of chiral symmetry restoration in the presence of massless fermions in the fundamental presentation. In nature quarks have small, but non-zero masses, which makes chiral symmetry only approximate. Nevertheless, the picture presented here still holds: confinement is driven by the dynamics of the chiral transition in the chiral limit. The argument can be extended even further: If quark masses were very large then chiral symmetry would be badly broken, and could not be used to characterize the phase transition. But in such a case the $Z_{2}$ symmetry becomes more exact, and by reversing the roles of the protagonists in the previous discussion, we would find that the $Z_{2}$ breaking drives the (approximate) restoration of chiral symmetry. Which of the underlying symmetries demands and which amends can be determined directly from the critical behavior of the spatial correlators of hadrons or of the Polyakov loop ${ }^{31,32}$. With quarks in the adjoint representation we investigated the physical scenario ${ }^{44}$ in which chiral symmetry is restored after deconfinement sets in. In this case we have pointed to the existence of an interesting structure, which was hidden until now: There are still two distinct phase transitions, but since the fields are now entangled, the transitions are not independent. This entanglement is shown at the level of expectation values and spatial correlators of the fields. More specifically, the spatial correlator of the field which is not at its critical temperature will in any case feel the phase transition measured by the other field. Lattice simulations will play an important role in checking these predictions. The results presented here are not limited to describing the chiral/deconfining phase transition and can readily be used to understand 
phase transitions sharing similar features.

The effective Lagrangians shown here can be immediately used, following ${ }^{24,30}$, to study the physics at RHIC. We expect the new trilinear term 32 , essential for our understanding of the relation between confinement and chiral symmetry breaking ${ }^{33}$, to play a role also for the physics of heavy ion collisions.

On a general level we have shown that the critical behavior encoded in the order parameter field is carried over the non-order parameter field. By monitoring spatial correlators not associated to the order parameter field one can determine the critical behavior of the theory.

\section{Acknowledgments}

We thank P. Damgaard, A. Jackson, J. Kapusta, J. Schechter and B. Tomasik for discussions and careful reading of the manuscript. We thank P. Petreczky and R. Pisarski for insightful discussions. F.S. thanks the organizers for having arranged such an interesting and stimulating conference.

\section{References}

1. F. Sannino, Phys. Lett. B 480, 280 (2000) [arXiv:hep-ph/0002277].

2. S. D. H. Hsu, F. Sannino and M. Schwetz, Mod. Phys. Lett. A 16, 1871 (2001) [arXiv:hep-ph/0006059].

3. F. Sannino, "Anomaly matching and low energy theories at high matter density," Proceedings for the conference on the Electroweak and Strong Matter, Heidelberg, 2001. arXiv:hep-ph/0301035.

4. T. Appelquist, Z. y. Duan and F. Sannino, Phys. Rev. D 61, 125009 (2000) [arXiv:hep-ph/0001043].

5. S. L. Adler and W. A. Bardeen, Phys. Rev. 182, 1517 (1969).

6. D. G. Sutherland, "Current Algebra And Some Nonstrong Mesonic Decays," Nucl. Phys. B 2, 433 (1967).

7. R. D. Pisarski, T. L. Trueman and M. H. G. Tytgat, Phys. Rev. D 56, 7077 (1997) [arXiv:hep-ph/9702362].

8. F. Sannino, Effective Lagrangians for QCD, Duality and Exact Results arXiv:hep-ph/0312375.

9. F. Sannino and M. Shifman, Effective Lagrangians for orientifold theories, arXiv:hep-th/0309252.

10. A. Armoni, M. Shifman and G. Veneziano, Phys. Rev. Lett. 91, 191601 (2003) [arXiv:hep-th/0307097].

11. M. Harada, F. Sannino and J. Schechter, arXiv:hep-ph/0309206. See also references therein related to the $\sigma$ particle.

12. M. Uehara, arXiv:hep-ph/0401037.

13. R. L. Jaffe, Phys. Rev. D 15, 267 (1977).

14. D. Black, A. H. Fariborz, F. Sannino and J. Schechter, Phys. Rev. D 59, 074026 (1999) [arXiv:hep-ph/9808415]. 
15. J. R. Pelaez, arXiv:hep-ph/0309292.

16. M. Uehara, arXiv:hep-ph/0308241.

17. E. Witten, Nucl. Phys. B 160, 57 (1979).

18. B. Lucini, M. Teper and U. Wenger, arXiv:hep-lat/0309170.

19. R. D. Pisarski and M. Tytgat, arXiv:hep-ph/9702340.

20. An incomplete list: A. M. Polyakov, Phys. Lett. B 72, 477 (1978); G. 't Hooft, Nucl. Phys. B 138, 1 (1978); A. Casher, Phys. Lett. B 83, 395 (1979); C. Adami, T. Hatsuda and I. Zahed, Phys. Rev. D 43, 921 (1991); G. E. Brown, A. D. Jackson, H. A. Bethe and P. M. Pizzochero, Nucl. Phys. A 560, 1035 (1993); F. Wilczek, Int. J. Mod. Phys. A 7, 3911 (1992) [Erratumibid. A 7, 6951 (1992)]; G. E. Brown, L. Grandchamp, C. H. Lee and M. Rho, arXiv:hep-ph/0308147; S. Digal, E. Laermann and H. Satz, Nucl. Phys. A 702, 159 (2002);

21. O.Scavenius, A.Mocsy, I.N.Mishustin and D.H.Rischke, Phys. Rev. C 64, 045202 (2001);

22. F. Sannino, N. Marchal and W. Schafer, Phys. Rev. D 66, 016007 (2002) [arXiv:hep-ph/0202248]; Y. Hatta and K. Fukushima, arXiv:hep-ph/0307068; K. Fukushima, arXiv:hep-ph/0310121.

23. F. Sannino, Phys. Rev. D 66, 034013 (2002) [arXiv:hep-ph/0204174].

24. A. Dumitru and R. D. Pisarski, Phys. Lett. B 504, 282 (2001) [arXiv:hep$\mathrm{ph} / 0010083$.

25. D. H. Rischke, arXiv:nucl-th/0305030.

26. B. Svetitsky and L. G. Yaffe, Nucl. Phys. B 210, 423 (1982)

27. L. G. Yaffe and B. Svetitsky, Phys. Rev. D 26, 963 (1982).

28. B. Svetitsky, Nucl. Phys. A 418, 477C (1984).

29. R. D. Pisarski, Phys. Rev. D 62, 111501 (2000) [arXiv:hep-ph/0006205].

30. O. Scavenius, A. Dumitru and J. T. Lenaghan, Phys. Rev. C 66, 034903 (2002) [arXiv:hep-ph/0201079]; O. Scavenius, A. Dumitru and A. D. Jackson, Phys. Rev. Lett. 87, 182302 (2001) [arXiv:hep-ph/0103219].

31. A. Mocsy, F. Sannino and K. Tuominen, Phys. Rev. Lett. 91, 092004 (2003) [arXiv:hep-ph/0301229].

32. A. Mocsy, F. Sannino and K. Tuominen, Induced universal properties and deconfinement, arXiv:hep-ph/0306069.

33. A. Mocsy, F. Sannino and K. Tuominen, Confinement versus chiral symmetry, arXiv:hep-ph/0308135.

34. J. Schechter, Phys. Rev. D 21, 3393 (1980); A. A. Migdal and M. A. Shifman, Phys. Lett. B 114, 445 (1982).

35. F. Sannino and J. Schechter, Phys. Rev. D 57, 170 (1998) [arXiv:hepth/9708113] and see references therein; F. Sannino and J. Schechter, Phys. Rev. D 60, 056004 (1999) [arXiv:hep-ph/9903359].

36. G. E. Brown and M. Rho, Phys. Rept. 363, 85 (2002) [arXiv:hep$\mathrm{ph} / 0103102]$.

37. N. O. Agasian, JETP Lett. 57 (1993) 208 [Pisma Zh. Eksp. Teor. Fiz. 57 (1993) 200].

38. G. W. Carter, O. Scavenius, I. N. Mishustin and P. J. Ellis, Phys. Rev. C 61, 045206 (2000) [arXiv:nucl-th/9812014]. 
39. N. O. Agasian and S. M. Fedorov, "Hadron resonance gas and nonperturbative QCD vacuum at finite temperature," arXiv:hep-ph/0310249.

40. A. Mocsy, F. Sannino and K. Tuominen, in preparation.

41. P. Bacilieri et al., Phys. Lett. B 220, 607 (1989).

42. S. Datta and S. Gupta, arXiv:hep-ph/9809382.

43. F. Karsch, Lect. Notes Phys. 583, 209 (2002) [arXiv:hep-lat/0106019].

44. F. Karsch and M. Lutgemeier, Nucl. Phys. B 550, 449 (1999) [arXiv:heplat/9812023].

45. B. Alles, M. D'Elia, M. P. Lombardo and M. Pepe, arXiv:hep-lat/0210039.

46. T. Banks and A. Ukawa, Nucl. Phys. B 225, 145 (1983).

47. F. Karsch, E. Laermann and A. Peikert, Nucl. Phys. B 605, 579 (2001) [arXiv:hep-lat/0012023].

48. A. Dumitru, D. Roder and J. Ruppert, arXiv:hep-ph/0311119.

49. E. V. Shuryak, Phys. Lett. B 107, 103 (1981).

50. R. D. Pisarski, Phys. Lett. B 110, 155 (1982).

51. A. Manohar and H. Georgi, Nucl. Phys. B 234, 189 (1984).

52. T. Appelquist, P. S. Rodrigues da Silva and F. Sannino, Phys. Rev. D 60, 116007 (1999). For the anomaly terms see: Z. Y. Duan, P. S. Rodrigues da Silva and F. Sannino, Nucl. Phys. B 592, 371 (2001) [arXiv:hep-ph/0001303].

53. J. T. Lenaghan, F. Sannino and K. Splittorff, Phys. Rev. D 65, 054002 (2002).

54. For a review on 2 color QCD see S. Hands, Nucl. Phys. Proc. Suppl. 106, $142(2002)$. 